\title{
Simulation of the dust flux on the ROSETTA probe during the orbiting phase around comet $46 \mathrm{P} /$ Wirtanen
}

\author{
M. Fulle ${ }^{1}$, L. Colangeli ${ }^{2}$, V. Mennella ${ }^{2}$, A. Rotundi ${ }^{3}$, and E. Bussoletti ${ }^{3}$ \\ 1 Osservatorio Astronomico di Trieste, via G.B. Tiepolo 11, I-34131 Trieste, Italy \\ 2 Osservatorio Astronomico di Capodimonte, Via Moiariello 16, I-80131 Napoli, Italy \\ ${ }^{3}$ Istituto di Fisica Sperimentale, Istituto Universitario Navale, Via A. De Gasperi 5, I-80133 Napoli, Italy
}

Received October 3, 1996; accepted February 7, 1997

\begin{abstract}
We present a probabilistic model of the dust mass, flux and fluence which will be collected by the ROSETTA probe while orbiting around comet $46 \mathrm{P} /$ Wirtanen. The dust environment of the target comet is simulated according to the most recent data available in the literature. Best fits of the DIDSY-GIOTTO data collected during the fly-by of comet $1 \mathrm{P} /$ Halley have shown that the probabilistic properties of dust ejection from the inner coma are crucial (Fulle et al. 1995). Therefore, we pay particular attention to the dust ejection velocity, which is assumed to have a wide distribution around the most probable values, and the dust ejection distribution, which is assumed to have a strong anisotropy peaked towards the sun. To compute the impact velocity in the probe reference frame, the rigorous keplerian orbit of each grain is considered taking into account aberrations due to the probe orbital velocity. We analyse the dependence of the results on the probe orbit parameters, such as true anomaly, probe-nucleus distance, orbit node and inclination. Computations are performed for the six main directions of the probe reference frame and for different values of the acceptance angle. The only way to collect direct grains is to point towards the nucleus; the mass collected in this direction is almost independent of the acceptance angle and of the time evolution of dust loss rate. A strong dependence of the collected dust mass on node and inclination is evidenced. By assuming an acceptance angle of $40^{\circ}$, the flux of reflected grains received in the two directions perpendicular to the probe orbit is higher than that in the nucleus direction, for $42 \%$ of randomly oriented probe orbits. The value increases up to $56 \%$ when the acceptance angle in the directions perpendicular to the probe orbit is increased up to $80^{\circ}$. The dust ejection anisotropy produces a strong dependence of the fluxes on the probe anomaly. For reflected grains, the fluences show relevant depletions at the largest masses, due to dust orbital effects, and the collected masses strongly depend on the acceptance angle
\end{abstract}

Send offprint requests to: M. Fulle and on the time evolution of the dust loss rate. The total dust fluxes are evaluated by assuming a half sphere field of view (corresponding to an acceptance angle of $180^{\circ}$ ).

Key words: Comets: general — Comets: individual: 46P/Wirtanen

\section{Introduction}

The ESA ROSETTA mission is planned to encounter comet 46P/Wirtanen after aphelion and to orbit around its nucleus while approaching the Sun (Bar-Nun et al. 1993). The probe orbit parameters will be very variable. The distance from the nucleus will range from thousands of $\mathrm{km}$, during the approaching phase, to less than $100 \mathrm{~km}$, during the mapping of the nucleus surface. In this latter phase, a complete surface sampling will be achieved by varying in a wide range the probe orbit angular parameters (i.e. inclination with respect to the comet orbital plane, pericenter argument and nodal line orientation with respect to the sun direction). Moreover, in the most probable case of an aspherical nucleus, the orbits will not be keplerian at the shortest probe-nucleus distances. Therefore, many orbit corrections might be necessary to maintain the probe in a cometocentric configuration. In order to constrain the required technical specifications for experiments included in the ROSETTA payload, the expected results must be simulated for all possible probe orbit configurations by assuming the most updated models of the $46 \mathrm{P} /$ Wirtanen nucleus. In the present paper, we model the dust flux expected on the Rosetta probe. The results will be primarily useful in the view of the dust flux analyser experiment, which is devoted to measuring the mass and velocity of the incoming dust. They will also be relevant both for the other experiments dealing with dust 
characterization and for those potentially affected by dust pollution.

So far, the only in-situ dust flux measurements were provided by the DIDSY experiment on-board the GIOTTO spacecraft (McDonnell et al. 1987). Fulle et al. (1995) obtained the best available fit of the DIDSY data by using a probabilistic model of the anisotropic dust ejection from $1 \mathrm{P} /$ Halley nucleus. Their analysis was focused on the unexpected millimetric grain excess observed in the collected fluence (i.e., the number of collected grains per unit area), a feature most likely simulated by a wide distribution of dust velocities. The adopted model included grains ejected over long times (from the GIOTTO fly-by back to the $1 \mathrm{P} /$ Halley perihelion) and covering a mass range from $310^{-12}$ to $510^{-5} \mathrm{~kg}$. The best fit of the DIDSY data provided the following values of the model parameters: a dust size distribution power index of $-3.5 \pm 0.2$; a dust velocity dispersion of $35 \pm$ $5 \mathrm{~m} \mathrm{~s}^{-1}$ around the most probable velocity, which agrees with predictions of hydrodynamic models for the 1P/Halley inner coma (Crifo 1991); a dust to gas ratio of $4 \pm 1$; an ejection dispersion of $18.4^{\circ}$ around the sun direction. Since information on the parameters required to model $46 \mathrm{P} /$ Wirtanen is mostly lacking, for our modelling we have adopted the above reported data. When possible, they have been updated with values provided by Jorda \& Rickman (1995), estimated from past visual and photographic observations of the $46 \mathrm{P} /$ Wirtanen coma, and with the dust ejection velocity values obtained by Crifo \& Rodionov (1996) on the basis of a model consistent with the photometric data quoted by Jorda \& Rickman (1995). The adopted parameters of the dust environment model of 46P/Wirtanen are summarized in Table 1.

Cometary dust is sensitive to the solar radiation pressure. Thus, for each probe position in the coma, two grain populations must be considered: those coming from the nucleus (hereafter direct grains) and those coming from the sun direction, under the action of the solar radiation pressure (hereafter reflected grains). The two populations are characterized by very different times of ejection from the nucleus. The most relevant difference between a fly-by (GIOTTO) and a rendez-vous (ROSETTA) configuration is that in the first case the probe velocity is always much higher than the dust velocities, so that the whole dust flux comes from the in front direction. On the contrary, the ROSETTA probe velocity will always be lower than the dust velocity, so that dust will impact the spacecraft from all directions. Thus, it becomes relevant to take into account the acceptance angle, $w$, of the considered experiment. The $w$ angle is the full aperture of the covered solid angle $2 \pi\left(1-\cos \frac{w}{2}\right)$.

In this paper we investigate: i) which fraction of dust flux will be collected by the instruments pointing towards the nucleus and characterized by a tight acceptance angle $w$; ii) which other view directions may offer useful dust flux sampling; iii) which fluences and dust masses are
Table 1. Adopted parameters for the dust environment of $46 \mathrm{P} /$ Wirtanen. $\quad \dot{m}_{\mathrm{q}}$, Perihelion gas loss rate. $\dot{m}(r)$, time-dependent gas loss rate. $r$, Sun-comet distance (AU). $v_{\mathrm{q}}$, Perihelion dust ejection velocity from the inner coma for micron-sized grains. $v_{\mathrm{d}}(r, s)$, time and size -dependent dust ejection velocity from the inner coma. $s$, dust diameter $(\mu \mathrm{m})$. $v_{\mathrm{e}}$, dust escape velocity from the nucleus surface. $v_{0}$, Dust velocity dispersion. $\phi_{0}$, dust ejection dispersion around the sun direction. $\chi$, dust to gas ratio. $\alpha$, dust size distribution power index. $s_{1}, s_{2}$, minimum and maximum dust sizes considered in the model. $\rho_{\mathrm{d}}, \rho_{\mathrm{n}}$, bulk densities of the dust and of the nucleus. $R_{\mathrm{n}}, M_{\mathrm{n}}$, Nucleus radius and mass

\begin{tabular}{cc}
\hline$\dot{m}_{\mathrm{q}}$ & $300 \mathrm{~kg} \mathrm{~s}^{-1}$ \\
$\dot{m}(r)$ & $\dot{m}_{\mathrm{q}} \mathrm{r}^{-6}$ \\
$v_{\mathrm{q}}$ & $300 \mathrm{~m} \mathrm{~s}^{-1}$ \\
$v_{\mathrm{d}}(r, s)$ & $v_{\mathrm{q}} \mathrm{r}^{-2} \mathrm{~s}^{-1 / 2}$ \\
$v_{\mathrm{e}}$ & $1 \mathrm{~m} \mathrm{~s}^{-1}$ \\
$v_{0}$ & $35 \mathrm{~m} \mathrm{~s}^{-1}$ \\
$\phi_{0}$ & $18.4^{\circ}$ \\
$\chi$ & 1 \\
$\alpha$ & -3.5 \\
$s_{1}$ & $210^{-5} \mathrm{~m}$ \\
$s_{2}$ & $810^{-3} \mathrm{~m}$ \\
$\rho_{\mathrm{d}}$ & $10^{3} \mathrm{~kg} \mathrm{~m}^{-3}$ \\
$\rho_{\mathrm{n}}$ & $10^{3} \mathrm{~kg} \mathrm{~m}^{-3}$ \\
$R_{\mathrm{n}}$ & $1.410^{3} \mathrm{~m}$ \\
$M_{\mathrm{n}}$ & $1.1310^{13} \mathrm{~kg}$ \\
\hline
\end{tabular}
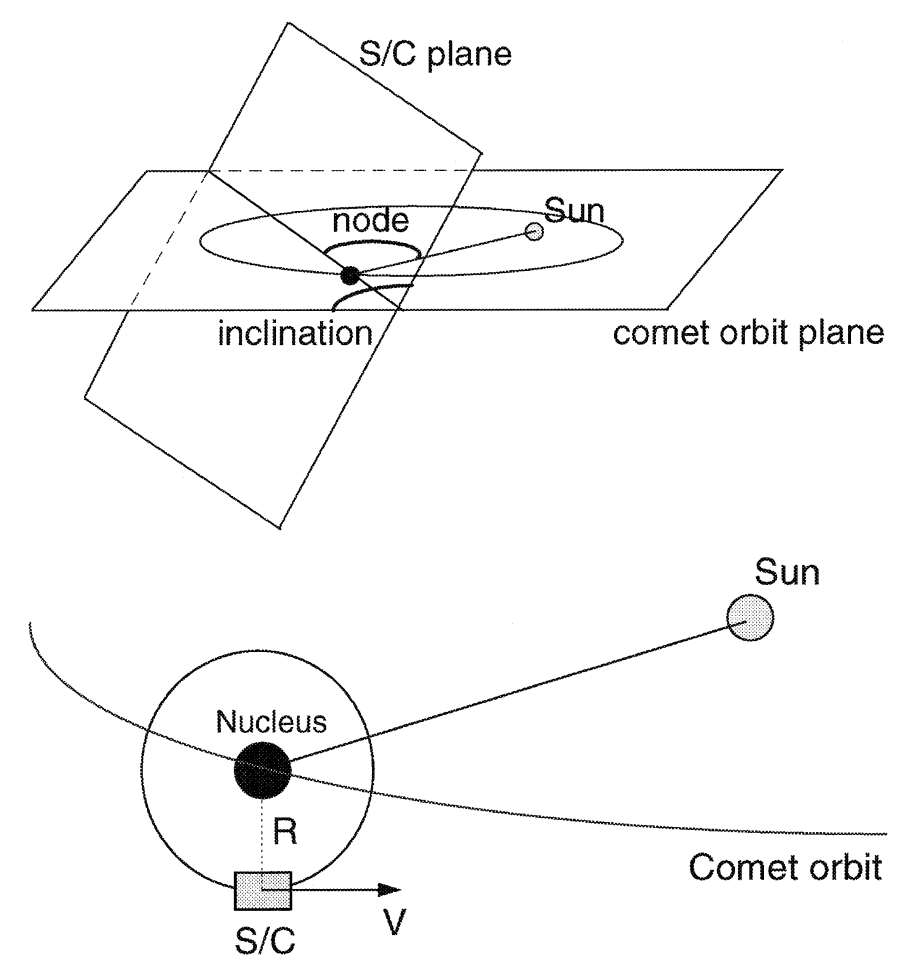

Fig. 1. Sketch of the ROSETTA orbital configuration 

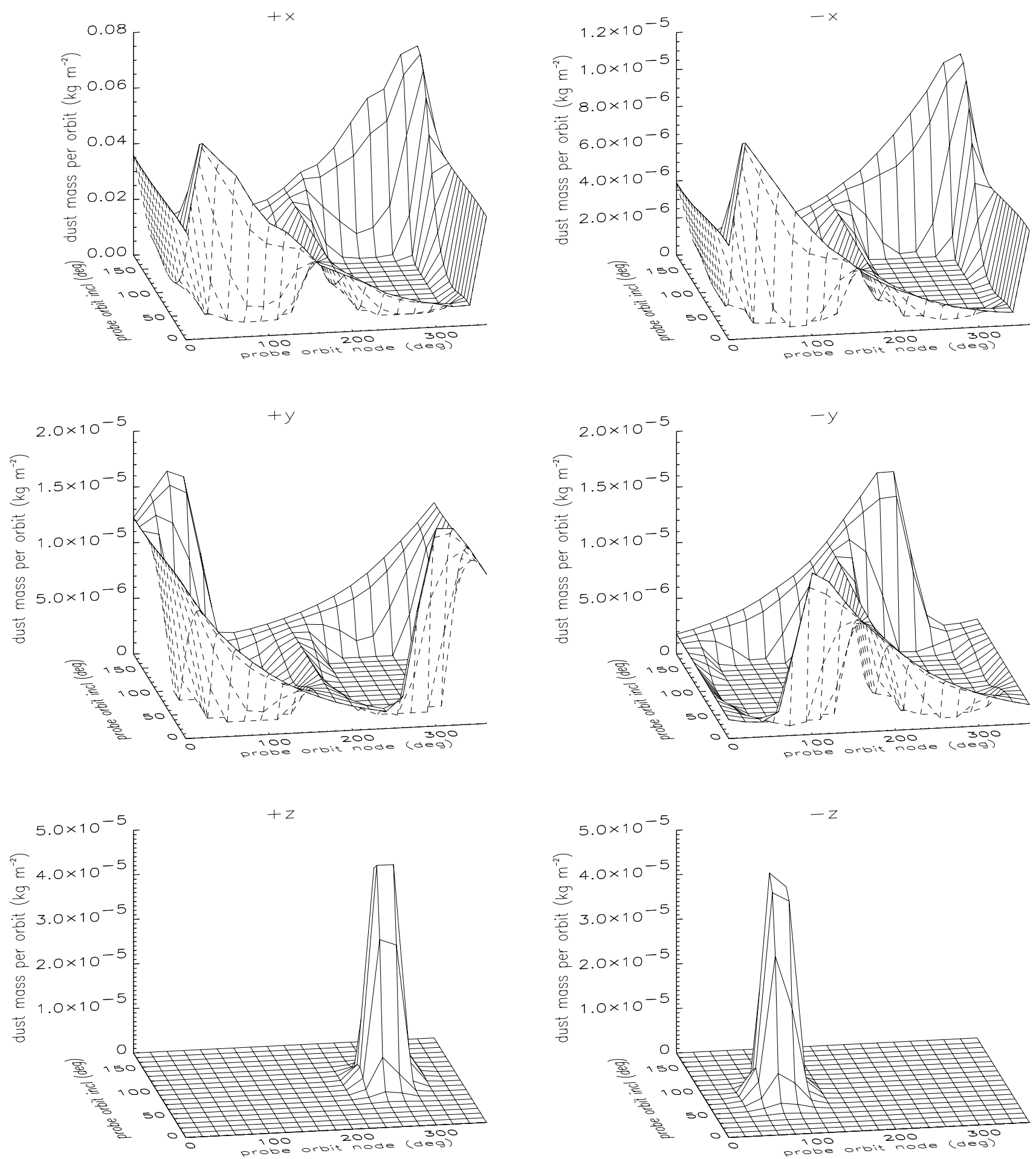

Fig. 2. Dust mass per unit surface collected during a probe orbit for $R=100 \mathrm{~km}$, starting sun-comet distance $r=1.762 \mathrm{AU}$, and $w=40^{\circ}$. The different panels refer to various pointing directions: comet nucleus direction $(+x)$ and its opposite $(-x)$; probe velocity vector direction $(+y)$ and its opposite $(-y)$; directions perpendicular to the probe orbital plane $(+z$ and $-z)$ 
expected to be collected for the comet environment parameters discussed above; iv) which is the dependence of all these quantities on $w$ and on the probe orbital parameters; v) which is the total dust flux on the orbiter, evaluated for $w=180^{\circ}$. Due to the large number of free parameters and the uncertainties affecting actual probe orbits, in this paper we assume circular orbits around the nucleus. Future works will have to analyse the dependence of the model results on the orbit eccentricity and on the variation of the parameters describing the dust environment of $46 \mathrm{P} /$ Wirtanen.
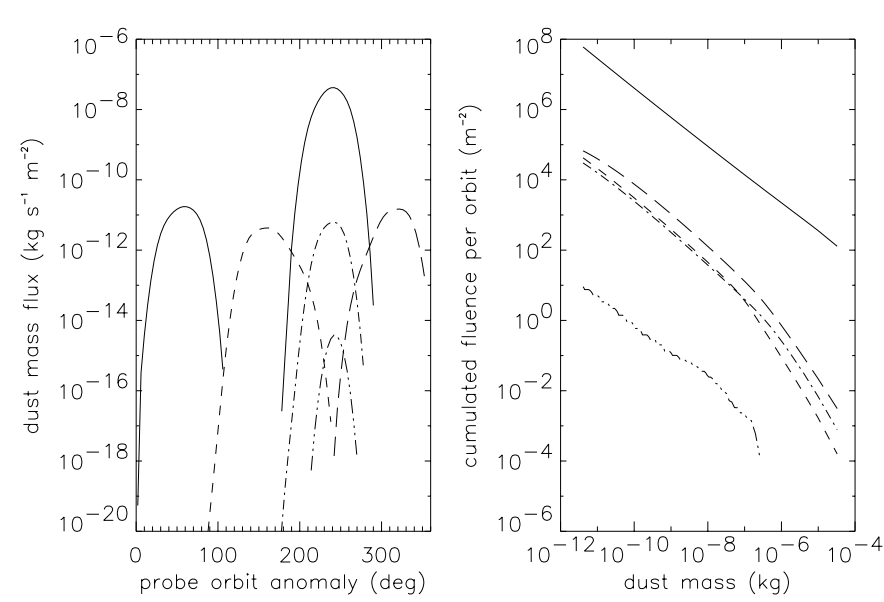

Fig. 3. Dust mass flux (left panel) and cumulated fluence (right panel) for $R=100 \mathrm{~km}$, starting sun-comet distance $r=1.762 \mathrm{AU}, w=40^{\circ}, n=120^{\circ}$ and $i=10^{\circ}$. The different line types refer to various pointing directions: $+x$ (continuous line), $-x$ (dot dashed line); $+y$ (short dashed line), $-y$ (long dashed line), and $-z$ (three dot dashed line)

\section{The model}

Fulle et al. (1995) have developed a probabilistic model able to estimate the dust flux on a probe, in a fly-by configuration (Fulle et al. 1995). To adapt the model to a rendez - vous scenario, the first relevant element to be considered is the similarity, in this case, of the probe and comet heliocentric velocities. The fly-by time scale is so short that changes occurring in the coma are negligible. On the contrary, the probe orbital period around the nucleus is generally so long that changes occurring in the coma, due to the variations of the sun comet distance, have to be accounted for. The main quantity measured by a dust collecting experiment is the dust fluence, i.e. the integral of the dust space density along the probe path. This is parametrized by the time, $t$, spent by the probe around the nucleus. Moreover, the dust space density in every point of the coma at a given time $t$ depends on the time integral of the dust ejection from the nucleus oc-
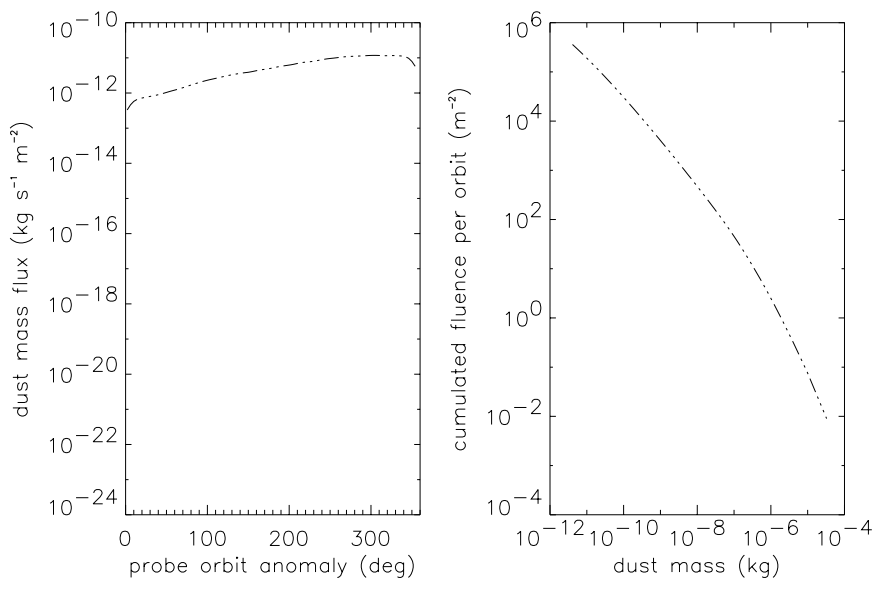

Fig. 4. Dust mass flux (left panel) and cumulated fluence (right panel) in the $-z$ direction for $R=100 \mathrm{~km}$, starting sun-comet distance $r=1.762 \mathrm{AU}, w=40^{\circ}, n=100^{\circ}$ and $i=90^{\circ}$
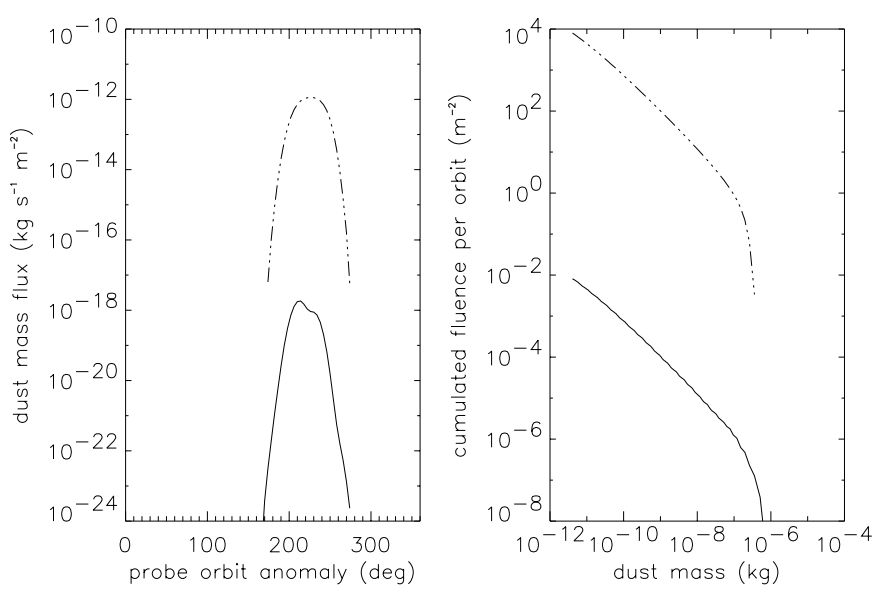

Fig. 5. Dust mass flux (left panel) and cumulated fluence (right panel) for $R=100 \mathrm{~km}$, starting sun-comet distance $r=1.762 \mathrm{AU}, w=40^{\circ}, n=120^{\circ}$ and $i=60^{\circ}$. Pointing directions: $+x$ (continuous line) and $-z$ (three dot dashed line)

curred in the past: this past history is parametrized by the time $\tau$, elapsed from dust ejection from the nucleus. It follows that the fluence comes from a double time integration. Thus, the time interval between ejection and impact on the probe is $t-\tau$. If we are interested in the dust fluence collected by the probe during one orbit, the time $t$ ranges between the starting time $t_{\mathrm{i}}$ and $t_{\mathrm{i}}+P$, where $P$ is the probe orbital period, while $\tau$ ranges from $\tau_{\mathrm{i}}$, corresponding to the comet aphelion (when the comet is assumed to be inactive) to the impact time $t$.

A fundamental quantity is the velocity vector of dust ejected from the inner coma that is required to impact the probe, $\mathbf{v}_{\mathbf{i}}(\tau, s, t)$. It depends on the dust ejection time, 

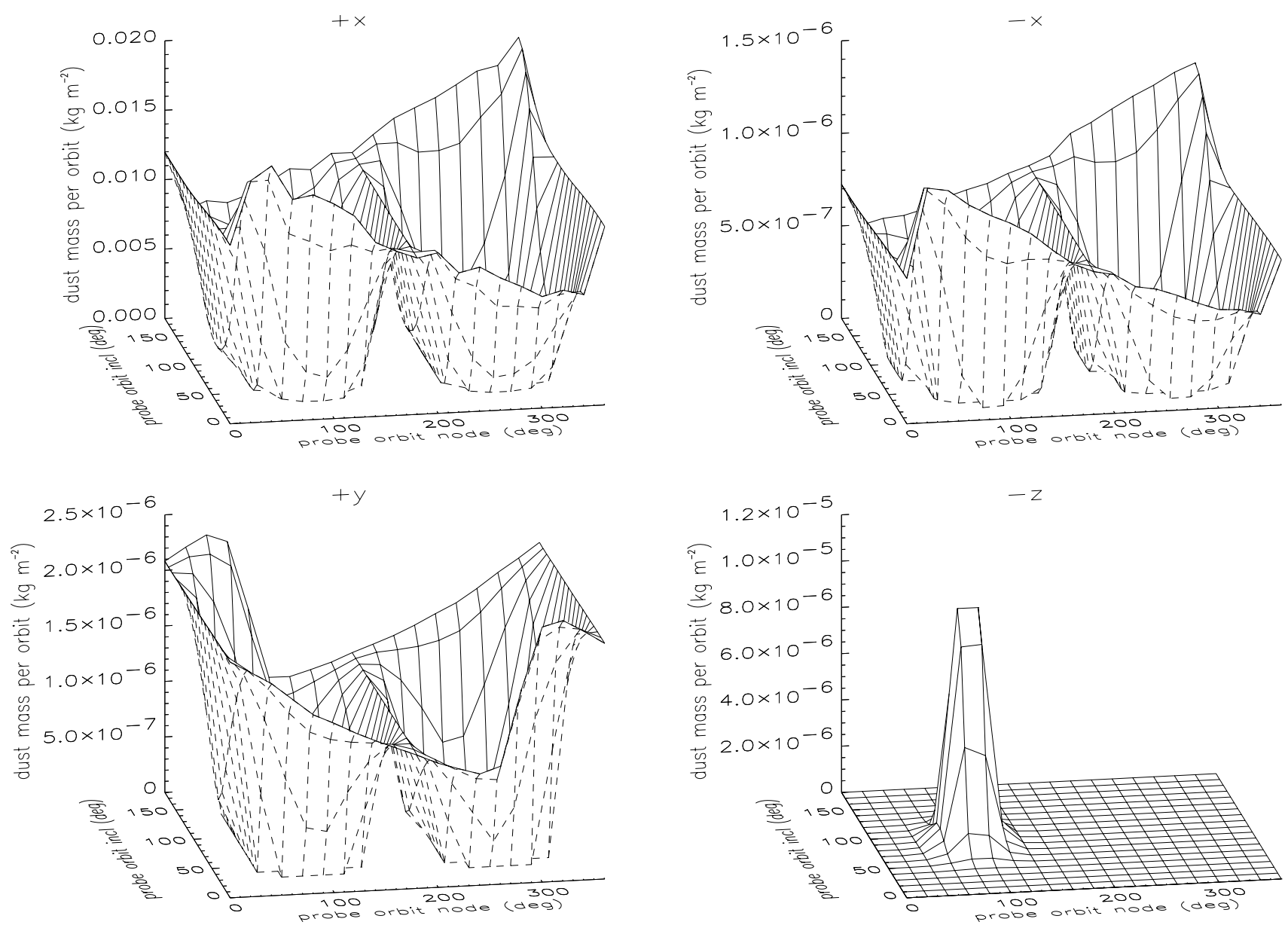

Fig. 6. Dust mass per unit surface collected during a probe orbit for $R=50 \mathrm{~km}$, starting sun-comet distance $r=1.762 \mathrm{AU}$, $w=40^{\circ}$. Pointing directions: $+x,-x,+y$ and $-z$

$\tau$, the grain diameter, $s$, and the probe position along its orbit, which is determined by $t$ and by the probe orbit geometry. Details for the computation of this vector were described by Fulle et al. (1995). By means of keplerian mechanics, from $\mathbf{v}_{\mathbf{i}}$ it is possible to compute the impact velocity vector projected in a probe reference frame, $\mathbf{v}_{\mathbf{f}}$. Dealing with $\mathbf{v}_{\mathbf{f}}$ we face a second relevant difference with respect to a fly-by scenario. In this case, $\mathbf{v}_{\mathbf{f}}$ results always opposite to the probe motion and its absolute value is equal to the probe velocity, which is much higher then the dust velocity. In the case of an orbiting probe, such a trivial case never occurs and $\mathbf{v}_{\mathbf{f}}$ can be computed from the vectorial sum of the negative probe orbital velocity and the dust impact velocity. Among the grains impacting the probe, only those having a vector $\mathbf{v}_{\mathbf{f}}$ entering the acceptance angle of the dust collecting experiment contribute to the measured dust fluence.

The aim of our dust flux model is to compute the differential fluence, $f(s)$, the cumulative fluence, $h(m)$, the total mass collected per unit surface, $\mathcal{M}$, and the mass flux, $\psi(t)$. Thus, we have to consider $\mathrm{d} N=$
$\dot{N}(\tau) g(s) p\left[\mathbf{v}_{\mathbf{i}}(\tau, s, t)\right] \mathrm{d} \tau \mathrm{d} s \mathrm{~d} v_{i}$, i.e. the number of particles inside the coma volume $\mathrm{d} V=\Phi r^{2} \mathrm{~d} r$. Here, $\dot{N}(\tau)$ is the grain number loss rate of the comet, $g(s)$ is the differential dust size distribution at the ejection and $p\left(\mathbf{v}_{\mathbf{i}}\right)$ represents the ejection velocity vector distribution (which describes both the velocity absolute values distribution and the ejection anisotropies) of grains ejected inside the solid angle $\Phi$. The length $r=(t-\tau) v_{\mathrm{i}}$ is the radius of the dust shell ejected with velocity $v_{\mathrm{i}}$, for time intervals between ejection $(\tau)$ and impact $(t)$ times - corresponding to anomalies smaller than $\frac{\pi}{2}$ (Finson \& Probstein approximation, 1968). The differential fluence, $f(s)$, is given by:

$f(s) \mathrm{d} s=$

$\int_{t_{\mathrm{i}}}^{t_{\mathrm{i}}+P} \int_{\tau_{\mathrm{i}}}^{t} \frac{\dot{N}(\tau) g(s) p\left[\mathbf{v}_{\mathrm{i}}(\tau, s, t)\right]}{\Phi v_{\mathrm{i}}^{3}(\tau, s, t)(t-\tau)^{2}}\left|\frac{\mathrm{d} v_{\mathrm{i}}(\tau, s, t)}{\mathrm{d} \tau}\right| v_{\mathrm{f}} \mathrm{d} t \mathrm{~d} \tau \mathrm{d} s(1)$

Here, the integration variable $v_{\mathrm{i}}$ is replaced by $\tau$ and $v_{\mathrm{f}}$ is the absolute value of the vector $\mathbf{v}_{\mathbf{f}}$, if this vector enters the acceptance angle of the experiment, otherwise $v_{\mathrm{f}}=0$. From the differential fluence, we obtain: 
Table 2. Values of parameters adopted in the simulations. $r$, Sun-comet distance. $R$, probe-nucleus distance. $P$, probe orbital period. $w$, view angle. $n, i$, probe orbit node and inclination. Fig. No, figure number

\begin{tabular}{ccccccc}
\hline$r(A U)$ & $R(\mathrm{~km})$ & $P(\mathrm{~d})$ & $w\left(^{\circ}\right)$ & $n\left(^{\circ}\right)$ & $i\left(^{\circ}\right)$ & Fig. No \\
\hline 1.762 & 100 & 91 & 40 & - & - & 2 \\
1.762 & 100 & 91 & 40 & 120 & 10 & 3 \\
1.762 & 100 & 91 & 40 & 100 & 90 & 4 \\
1.762 & 100 & 91 & 40 & 120 & 60 & 5 \\
1.762 & 50 & 32 & 40 & - & - & 6 \\
1.762 & 50 & 32 & 40 & 120 & 10 & 7 \\
2.624 & 100 & 91 & 40 & - & - & 8 \\
2.624 & 100 & 91 & 40 & 120 & 10 & 9 \\
1.762 & 100 & 91 & 80 & - & - & 10 \\
1.762 & 100 & 91 & 80 & 120 & 10 & 11 \\
1.762 & 100 & 91 & 80 & 120 & 60 & 12 \\
1.762 & 100 & 91 & 180 & - & - & 13 \\
1.762 & 100 & 91 & 180 & 120 & 10 & 14 \\
1.762 & 100 & 91 & 180 & 100 & 90 & 15 \\
1.762 & 100 & 91 & 180 & 120 & 60 & 16 \\
1.762 & 50 & 32 & 180 & - & - & 17 \\
1.762 & 50 & 32 & 180 & 120 & 10 & 18 \\
2.624 & 100 & 91 & 180 & - & - & 19 \\
2.624 & 100 & 91 & 180 & 120 & 10 & 20 \\
\hline
\end{tabular}

(i) the dust mass per unit surface collected in a probe orbit

$\mathcal{M}=\frac{\pi}{6} \rho_{\mathrm{d}} \int_{s_{1}}^{s_{2}} s^{3} f(s) \mathrm{d} s$

(ii) the cumulated fluence per unit surface, collected in a probe orbit

$h(m)=\int_{s(m)}^{s\left(m_{2}\right)} f(s) \mathrm{d} s$

(iii) the dust mass flux per unit time and surface, collected in a probe orbit

$\psi(t)=\frac{\pi}{6} \rho_{\mathrm{d}} \int_{s_{1}}^{s_{2}} s^{3} \frac{\mathrm{d} f(s)}{\mathrm{d} t} \mathrm{~d} s$

where $s_{1}=210^{-5} \mathrm{~m}, s_{2}=810^{-3} \mathrm{~m}$ and $\rho_{\mathrm{d}}=$ $10^{3} \mathrm{~kg} \mathrm{~m}^{-3}$. For $p\left(\mathbf{v}_{\mathbf{i}}\right)$, we assume the following gaussian distributions:

$p(v)=\frac{2 \exp -\frac{[v-v(\tau, s)]^{2}}{v_{0}^{2}}}{v_{0} \sqrt{\pi}\left[1+\operatorname{erf} \frac{v(\tau, s)}{v_{0}}\right]}$

$\frac{p[\phi(v)]}{\Phi}=\frac{\exp -\frac{[1-\cos \phi(v)]^{2}}{\left(1-\cos \phi_{0}\right)^{2}}}{\pi^{3 / 2}\left(1-\cos \phi_{0}\right) e r f \frac{2}{1-\cos \phi_{0}}}$

where $\operatorname{erf}$ is the error function, $v(\tau, s)$ is the most probable velocity, $v_{0}$ is the velocity dispersion, $\phi(v)$ is the solar
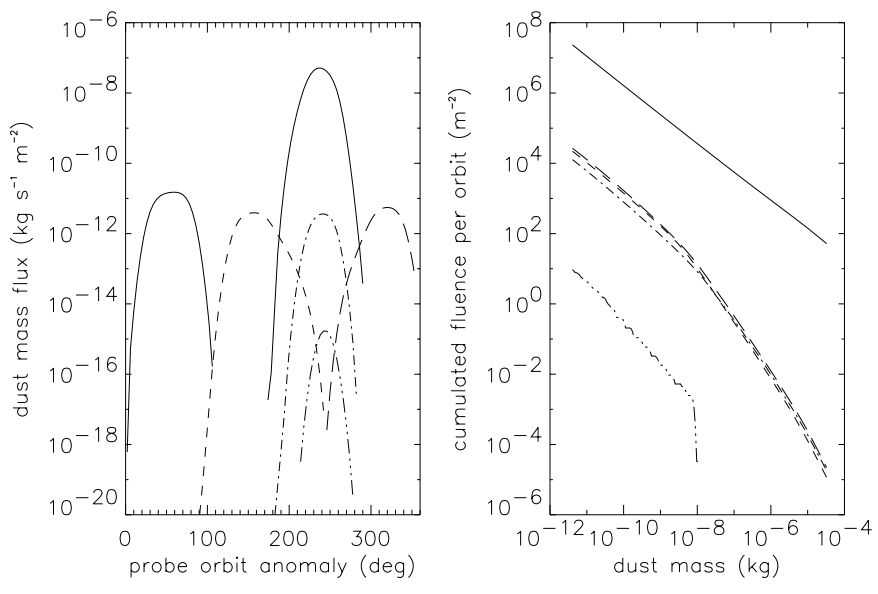

Fig. 7. Dust mass flux (left panel) and cumulated fluence (right panel) for $R=50 \mathrm{~km}$, starting sun-comet distance $r=1.762 \mathrm{AU}, w=40^{\circ}, n=120^{\circ}$ and $i=10^{\circ}$. Pointing directions: $+x$ (continuous line), $-x$ (dot dashed line), $+y$ (short dashed line), $-y$ (long dashed line), and $-z$ (three dot dashed line)

zenithal angle and $\phi_{0}$ is the ejection dispersion. Finally, the gas loss rate and the dust to gas ratio allow us to compute the dust mass loss rate and, then, $\dot{N}(\tau)$ during the comet orbit by means of $g(s)$.

\section{Results}

In Figs. 1a, b the ROSETTA orbit configuration is sketched. The inclination, $i$, is defined as the angle between the probe and the comet orbital planes; the node, $n$, is defined as the angle between the comet-sun vector and the probe orbit ascending nodal line. The node is zero when the probe ascending node is between the sun and the comet nucleus, and corotates with the comet orbit anomaly. The probe orbit anomaly starts from the ascending node and corotates (for prograde orbits) with the comet anomaly. The results here reported are obtained assuming the comet environment parameters listed in Table 1 and refer to different orbit configurations (i.e. sun-comet distance, probe-nucleus distance, probe orbit node and inclination) and various acceptance angles, $w$. The considered cases are summarized in Table 2, where reference is also given to the figures relevant for each combination of parameters. For the adopted parameters, the probe orbital period, $P$, is quite long, so that the sun-comet distance significantly changes during one probe orbit. A long $P$ is determined by the small nucleus mass and would further increase for values of the bulk nucleus density and of the nucleus radius lower than the assumed $10^{3} \mathrm{~kg} \mathrm{~m}^{-3}$ and $1.4 \mathrm{~km}$, respectively.

In Fig. 2 we plot the $\mathcal{M}$ values for all the possible probe orbit orientations in the case $r=1.762 \mathrm{AU}, R=100 \mathrm{~km}$ and $w=40^{\circ}$. The main viewing directions in the probe 

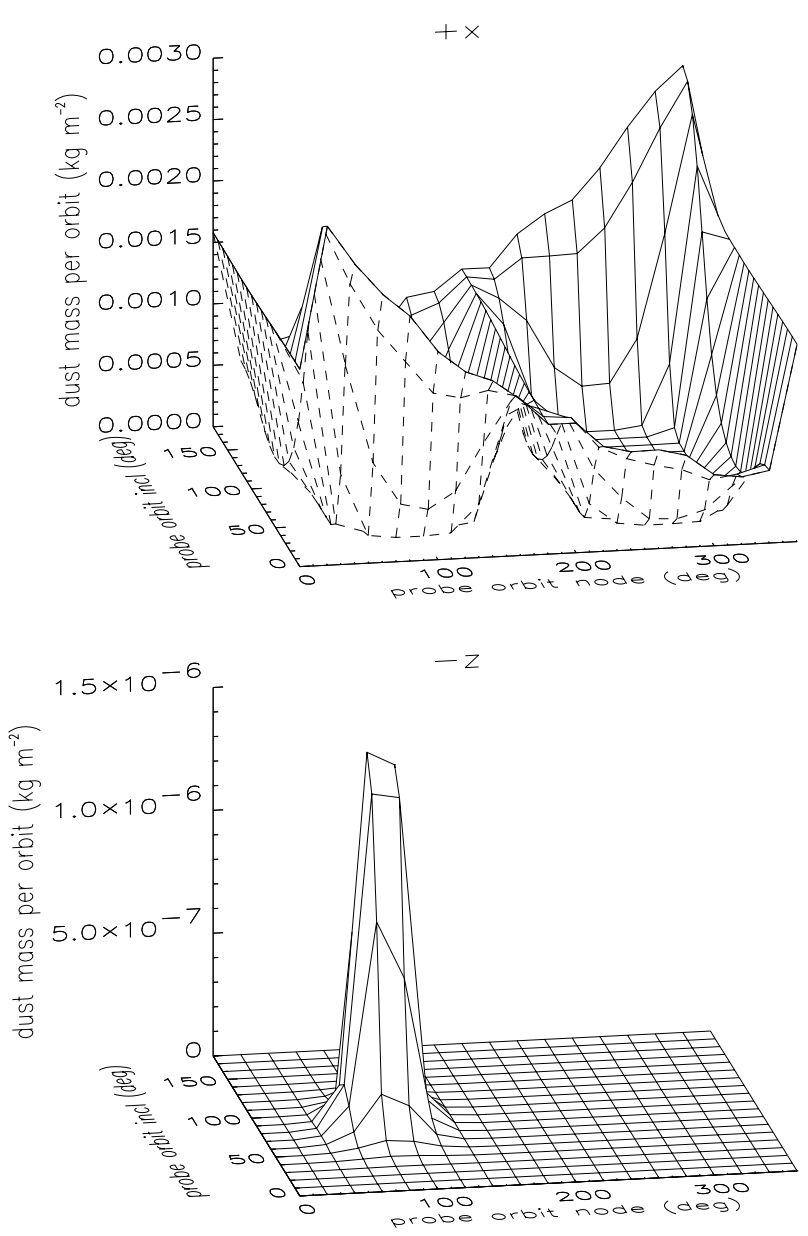

Fig. 8. Dust mass per unit surface collected during a probe orbit for $R=100 \mathrm{~km}$, starting sun-comet distance $r=$ $2.624 \mathrm{AU}, w=40^{\circ}$. Pointing directions: $+x$ and $-z$

reference frame have been considered: the nucleus direction $(+x)$, the velocity vector $(+y)$, the direction perpendicular to the probe orbital plane $(+z)$ and the corresponding opposite directions $(-x,-y,-z)$. Within a few percent variation, an evident symmetry is observed between prograde $\left(i<90^{\circ}\right)$ and retrograde $\left(i>90^{\circ}\right)$ orbits and between orbital nodes $n<180^{\circ}$ and $n>180^{\circ}$. This is a consequence of the coma axial symmetry, which, however, is not perfect due to the keplerian motion of the dust grains. For the same reason, the plots in the $+y$ and $+z$ directions closely resemble those in the $-y$ and $-z$ directions, respectively, after a node shift of $180^{\circ}$. Therefore, in order to sample the dependence of the collected dust flux on the orbital parameters, it is sufficient to consider the directions $+x,-x,+y$ and $-z$ in the node range between $0^{\circ}$ and $180^{\circ}$.

The plots evidence the complexity of the dust mass dependence on the probe orbital parameters, in the most probable case of a strongly anisotropic dust ejection from the inner coma. The largest masses are found in the $+x$
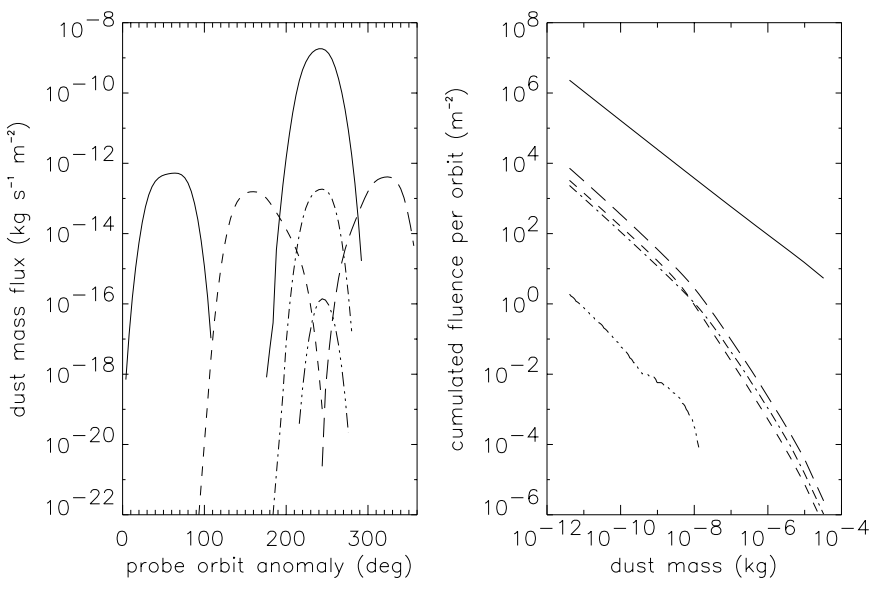

Fig. 9. Dust mass flux (left panel) and cumulated fluence (right panel) for $R=100 \mathrm{~km}$, starting sun-comet distance $r=2.624 \mathrm{AU}, w=40^{\circ}, n=120^{\circ}$ and $i=10^{\circ}$. Pointing directions: $+x$ (continuous line), $-x$ (dot dashed line), $+y$ (short dashed line), $-y$ (long dashed line), and $-z$ (three dot dashed line)

direction, as direct grains are collected only in this case. We recall that their space density is much higher than that of reflected particles. In a first order approximation, the dust path from the nucleus to the probe is a parabola. This is due to the solar radiation pressure acting on the grains, which is roughly independent of the probe-nucleus distance. Therefore, two dust paths are in general possible for each probe position, the direct one, with higher ejection solar zenithal angle and shorter flight time, and the reflected one, with lower ejection solar zenithal angle and longer flight time. It follows that direct grains come approximately from the nucleus, whereas reflected ones from the sun direction. For a probe orbit node of $0^{\circ}$ or $180^{\circ}$, the collected mass does not depend significantly on the inclination, since the probe always crosses the sun-pointing jet. For other node values, the largest mass is collected when the probe crosses the jet at the smallest sun-comet distance; this happens for a node value close to the ejection dispersion. For the corresponding negative node value the smallest mass is collected, as the probe crosses the jet at the largest sun-comet distance. Finally, for nodes and inclinations close to $90^{\circ}$, the probe never crosses the jet, so that no dust is collected from the $+x$ direction.

The dust mass from the $-x$ direction (Fig. 2) is due to reflected grains and, as in the previous case, is collected mostly when the probe is between comet and sun. Thus, it shows a very similar dependence on the probe orbit angles and much lower absolute values. The mass from the $+y$ direction shows a similar behaviour, after a proper shift of the nodes, as again it is due to reflected grains. The collected mass is higher than in the $-x$ direction. In fact, most of the total dust in the $+y$ direction is collected in 

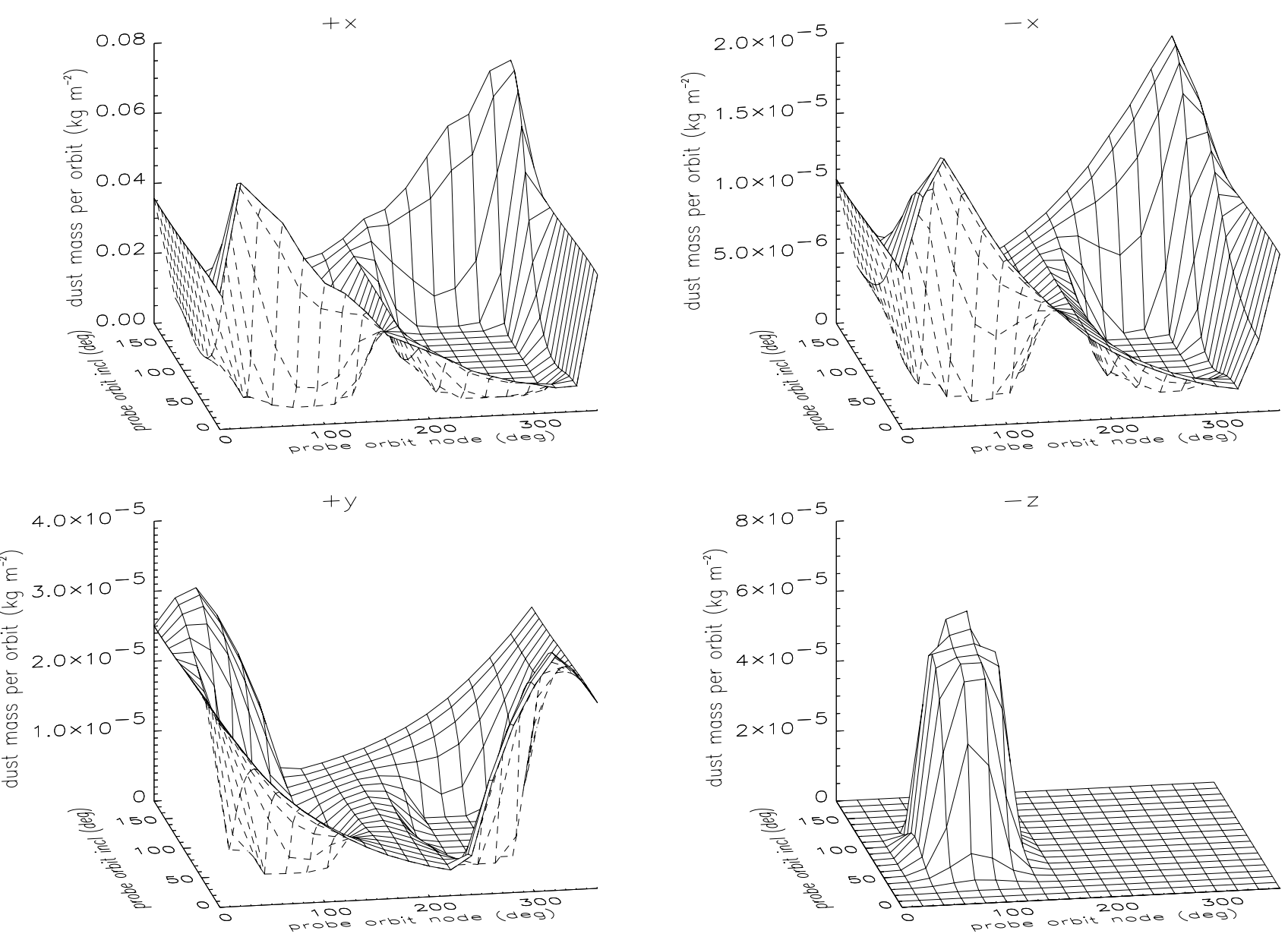

Fig. 10. Dust mass per unit surface collected during a probe orbit for $R=100 \mathrm{~km}$, starting sun-comet distance $r=1.762 \mathrm{AU}$, $w=80^{\circ}$. Pointing directions: $+x,-x,+y$ and $-z$

the orbit position where the probe velocity points to the sun. Here, the grain space density is higher than in the region between comet and sun. As far as the mass collected from $+z$ direction is concerned, a completely different dependence on the orbit angles results, as dust is collected for such probe orbit angles that no grains are received for all the other directions. From a statistical analysis it comes out that in the $42 \%$ of randomly oriented probe orbits, dust mass collected in the $+z$ and $-z$ directions is larger than in the $+x$ direction. Moreover, in all these favourable cases, reflected grains are collected during the whole orbit in the $+z$ and $-z$ directions. These directions appear the most appropriate to collect the largest mass of reflected grains.

Figures 3, 4 and 5 show the fluxes, $\psi(t)$, and fluences, $h(m)$, for selected values of the probe orbit angles. Figure 3 shows a typical case of low inclination orbit, for which the $+x$ direction provides the largest flux. This direction points towards the sun for a probe anomaly of $60^{\circ}$, so that it collects reflected grains, whereas for $240^{\circ}$ it collects direct grains. For this last anomaly, the $-x$ and the $+z$ directions collect reflected grains: despite the small inclination, the probe moves well below the comet orbital plane, so that the $+z$ direction can collect grains reflected out of the comet orbital plane. The largest reflected flux in the $+x$ direction is due to the most favourable combination of sun-comet and sun-probe distances. The fluences reported in Fig. 3 evidence the changes introduced by the dust keplerian orbits on the power index of the dust size distribution: only direct fluences maintain a constant power index; the reflected fluences are depleted of the largest grains. Figure 3 is representative of all the cases for which the highest flux is due to direct grains. A case favourable to dust collection in the $+z$ direction is reported in Fig. 4. The increasing flux is due to the decreasing sun-comet distance. Figure 5 shows that, even in a less favourable case, the flux from the $+z$ direction is orders of magnitude higher than from the $+x$ direction. The fluences in both directions are depleted in the largest grains, because both refer to reflected grains.

To analyse the dependence of the model results on the probe orbit radius, $R$, in Fig. 6 we plot the $\mathcal{M}$ values for 

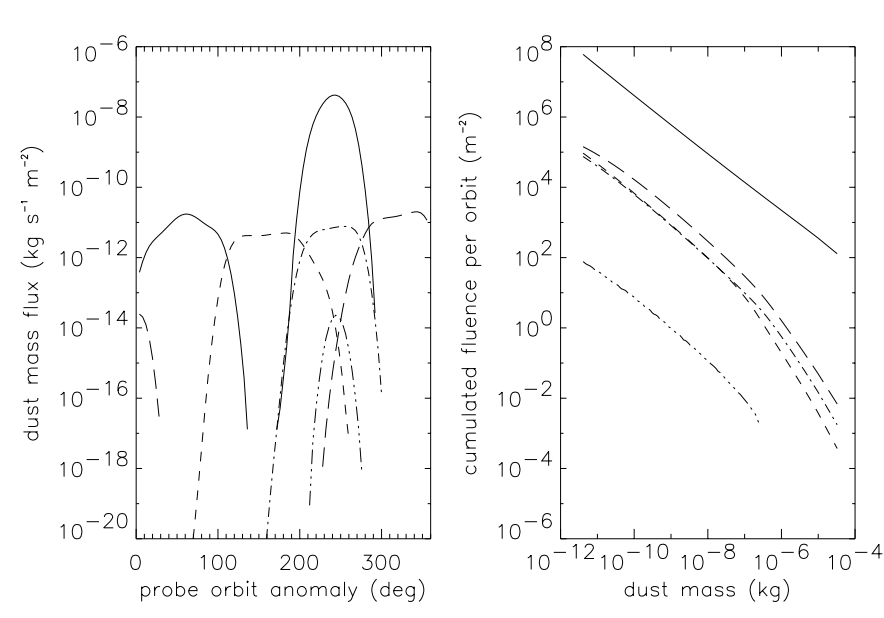

Fig. 11. Dust mass flux (left panel) and cumulated fluence (right panel) for $R=100 \mathrm{~km}$, starting sun-comet distance $r=1.762 \mathrm{AU}, w=80^{\circ}, n=120^{\circ}$ and $i=10^{\circ}$. Pointing directions: $+x$ (continuous line), $-x$ (dot dashed line),$+y$ (short dashed line), $-y$ (long dashed line), and $-z$ (three dot dashed line)
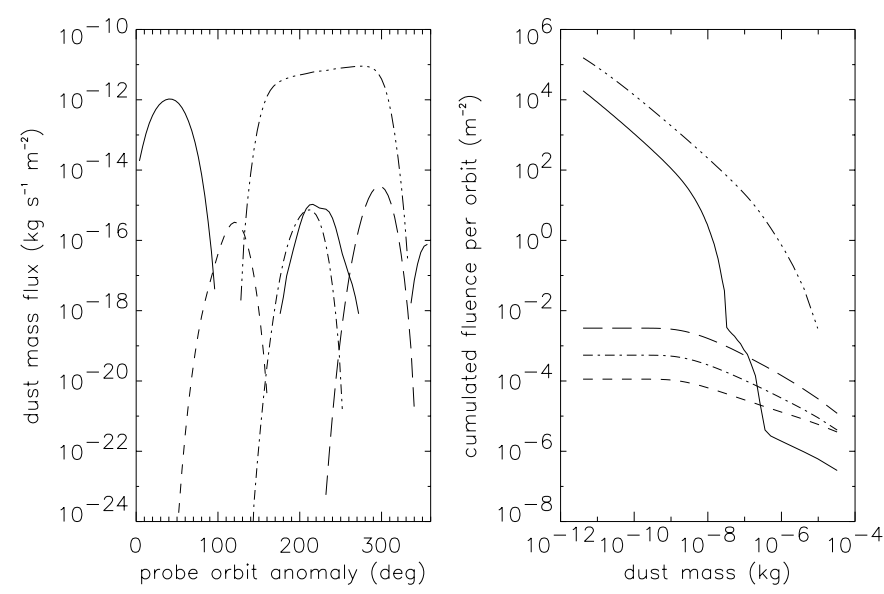

Fig. 12. Dust mass flux (left panel) and cumulated fluence (right panel) for $R=100 \mathrm{~km}$, starting sun-comet distance $r=1.762 \mathrm{AU}, w=80^{\circ}, n=120^{\circ}$ and $i=60^{\circ}$. Pointing directions: $+x$ (continuous line), $-x$ (dot dashed line), $+y$ (short dashed line), $-y$ (long dashed line), and $-z$ (three dot dashed line)

the same parameters as before, but $R=50 \mathrm{~km}$. This is a lower limit of the model for the considered comet environment. Indeed, at shorter $R$ the probe should fall in the gas drag region. The $R$ decrease produces a less pronounced mass dependence on the probe orbit node, as smaller changes of the dust loss rate are associated with a shorter period. Moreover, we find lower mass values, even if larger fluxes are expected closer to the nucleus. To reconcile this apparent discrepancy, we have to consider that the plotted masses are integrated over a shorter period,
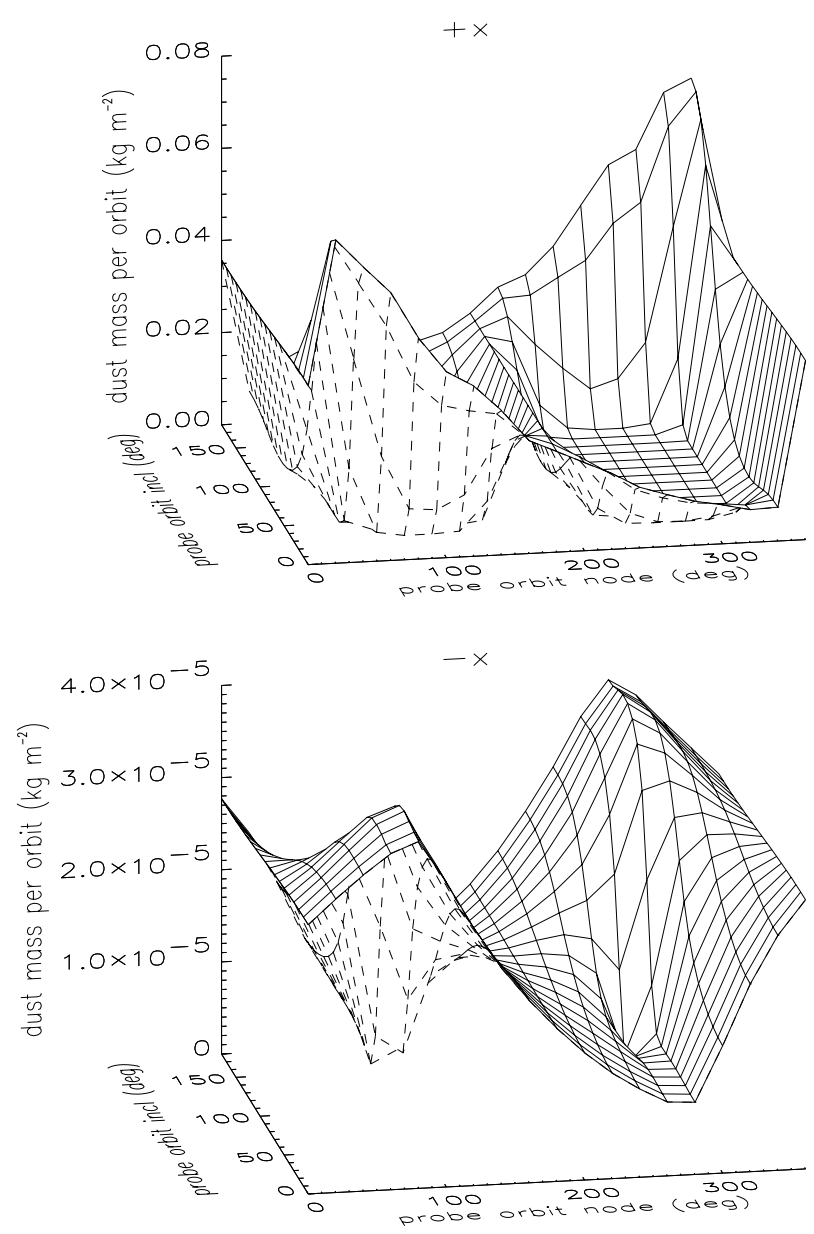

Fig. 13. Dust mass per unit surface collected during a probe orbit for $R=100 \mathrm{~km}$, starting sun-comet distance $r=1.762$ $\mathrm{AU}, w=180^{\circ}$. Pointing directions: $+x$ and $-x$

the mean sun-comet distance is larger and the dust loss rate is lower than for the $R=100 \mathrm{~km}$ case. We confirm that the mass collected in the $+z$ and $-z$ directions is the highest for the $42 \%$ of randomly oriented probe orbits. The collected fluxes and fluences for $R=50 \mathrm{~km}$ show negligible differences with respect to those obtained for $R=100 \mathrm{~km}$. In Fig. 7 we report an example to be compared with Fig 3. For instance, the similarity of the main maxima due to direct grains is explained by the balance between larger sun-comet distance and higher dust space density at smaller $R$.

To explore the effects of starting sun-comet distance, $r$, we consider the case with $r=2.624$ AU: differences mainly regard the absolute values of the collected mass, flux and fluence. This result indicates that the dust loss rate is the most effective parameter, among those characterizing the comet environment. For sake of simplicity we report in Figs. 8 and 9 some relevant examples. We note that the fluences of the reflected grains have a cut-off at lower masses for larger $r$, where their number is much 

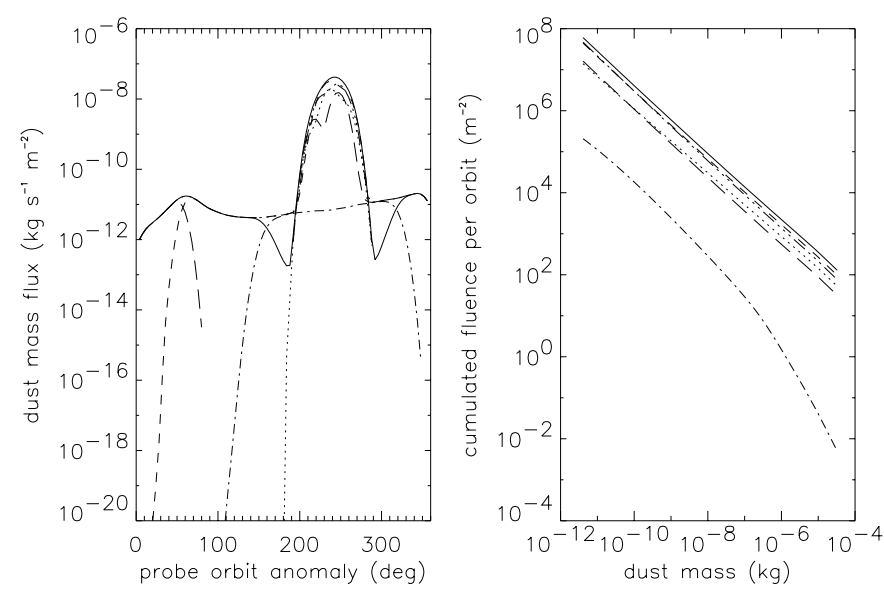

Fig. 14. Dust mass flux (left panel) and cumulated fluence (right panel) for $R=100 \mathrm{~km}$, starting sun-comet distance $r=1.762 \mathrm{AU}, w=180^{\circ}, n=120^{\circ}$ and $i=10^{\circ}$. The different line types refer to various pointing directions: $+x$ (continuous line), $-x$ (dot dashed line), $+y$ (short dashed line), $-y$ (long dashed line $),+z$ (dotted line), and $-z$ (three dot dashed line)
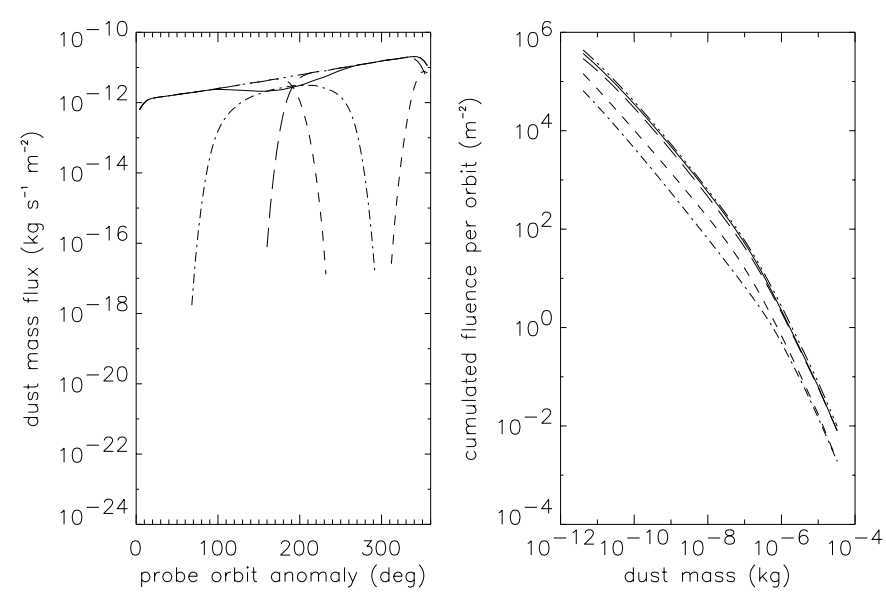

Fig. 15. Dust mass flux (left panel) and cumulated fluence (right panel) for $R=100 \mathrm{~km}$, starting sun-comet distance $r=1.762 \mathrm{AU}, w=180^{\circ}, n=100^{\circ}$ and $i=90^{\circ}$. The different line types refer to various pointing directions: $+x$ (continuous line), $-x$ (dot dashed line), $+y$ (short dashed line), $-y$ (long dashed line $),+z$ (dotted line), and $-z$ (three dot dashed line)

lower than close to the perihelion, due to the shorter time interval between aphelion and collection. It is worthwhile stressing that grains with masses larger than the cut-offs given by Crifo \& Rodionov (1996) can reach the probe due to the wide dust velocity distribution assumed in our model (see also Fulle et al. 1995). This wide distribution allows large grains to overcome the nucleus gravity even at large sun distances. Again, at large sun-comet distance, the dust mass collected from the $+z$ and $-z$ directions is
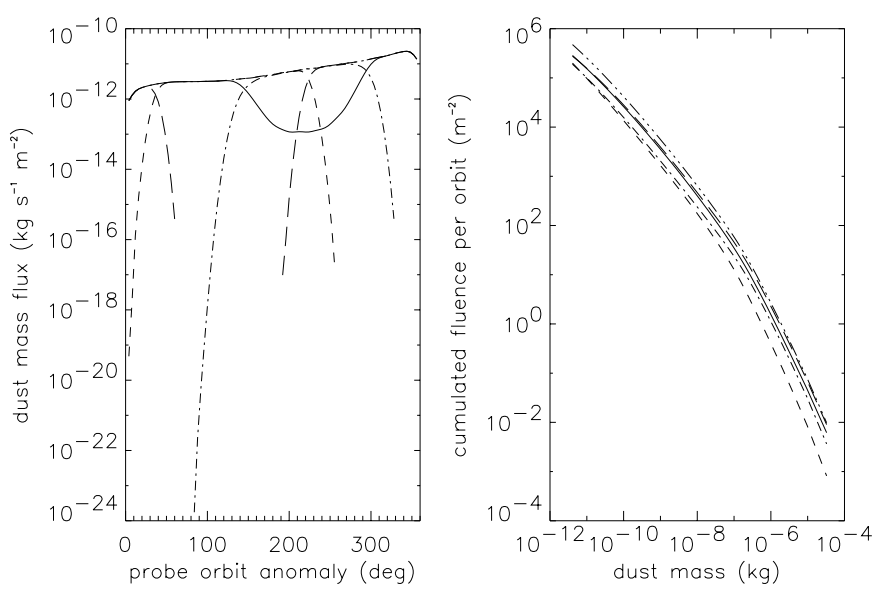

Fig. 16. Dust mass flux (left panel) and cumulated fluence (right panel) for $R=100 \mathrm{~km}$, starting sun-comet distance $r=1.762 \mathrm{AU}, w=180^{\circ}, n=120^{\circ}$ and $i=60^{\circ}$. The different line types refer to various pointing directions: $+x$ (continuous line), $-x$ (dot dashed line), $+y$ (short dashed line), $-y$ (long dashed line), $+z$ (dotted line), and $-z$ (three dot dashed line)

higher than that collected from the $+x$ direction for the $42 \%$ of randomly oriented probe orbits.

The case of a larger acceptance angle $\left(80^{\circ}\right)$ is considered in Fig. 10. The comparison with the results in Fig. 2 evidences that the flux of reflected grains increases, while the flux of direct ones is not affected. Since the reflected grains come from the sun direction, a larger view angle allows their collection for a larger fraction of the probe orbit. This effect is negligible in the $+x$ direction due to the dominance of the direct particles. Fluxes and fluences in Figs. 11 and 12 are consistent with this behaviour. A remarkable difference is the larger fluences of reflected grains in Fig. 11 with respect to Fig. 3. The comparison of Figs. 12 and 5 shows how the doubling of acceptance angle increases the flux of reflected grains in most of the considered directions. The highest increase of reflected grain flux is in the $+x$ direction. In fact, the flux from the $+z$ and $-z$ directions is higher than that from the $+x$ direction for $30 \%$ only of randomly oriented probe orbits.

The case of $w=180^{\circ}$ allows us to infer the global dust pollution on the probe. For this acceptance angle, the whole solid angle is covered by two view directions, so that we limit the plots of the total collected mass to the $+x$ and $-x$ directions. In this case, all the view directions collect direct and reflected grains, but the $-x$ direction, which collects reflected grains only. Figure 13 shows the $\mathcal{M}$ plots for the same parameters as for Fig. 2 (see Table 2 ). The comparison allows us to confirm that the whole flux of direct grains is received within an acceptance angle of $40^{\circ}(+x$ direction in Figs. 2 and 13). Figures $14-16$ show the fluxes and fluences for the same parameters as for Figs. 3-5 (see Table 2). The flux of direct grains does 

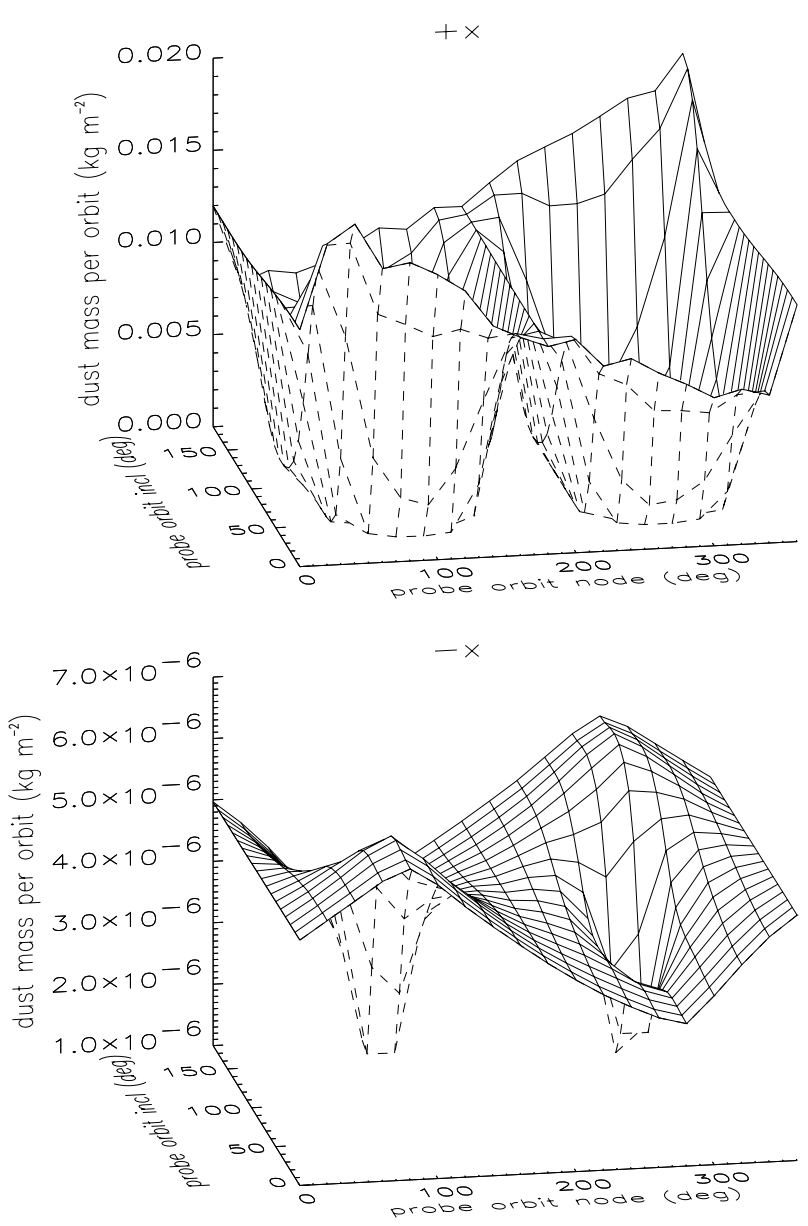

Fig. 17. Dust mass per unit surface collected during a probe orbit for $R=50 \mathrm{~km}$, starting sun-comet distance $r=$ $1.762 \mathrm{AU}, w=180^{\circ}$. Pointing directions: $+x$ and $-x$.

not increase for larger $w$, whereas the number of reflected grains collected from the $+x$ and $-x$ directions strongly increases, as well as the number of direct grains from the other directions. In particular, the fluxes from the $+y,-y$, $+z$ and $-z$ directions become similar to that from the $+x$ direction. The fluences are consistent with these conclusions and allow us to infer some information about the dust pollution of experiments facing the nucleus. Even in the unfavourable assumption that the whole fluence is due to the smallest grains (which produce the largest surface coverage), during the probe orbit period about $1 \%$ only of the exposed area would be covered by direct grains. It follows that the first dust monolayer would be reached after many probe orbits similar to those considered here. The results in Figs. 17 - 20, which refer to the same parameter combinations of Figs. 6 - 9 (Table 2), allow us to extend the conclusions reported above to the other considered sets of parameters.
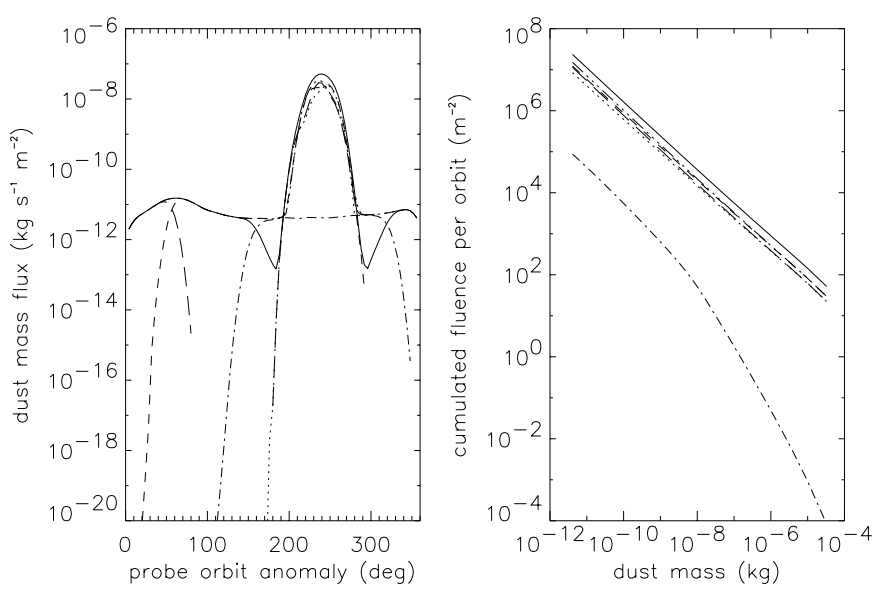

Fig. 18. Dust mass flux (left panel) and cumulated fluence (right panel) for $R=50 \mathrm{~km}$, starting sun-comet distance $r=1.762 \mathrm{AU}, w=180^{\circ}, n=120^{\circ}$ and $i=10^{\circ}$. Pointing directions: $+x$ (continuous line), $-x$ (dot dashed line), $+y$ (short dashed line), $-y$ (long dashed line) $+z$ (dotted line), and $-z$ (three dot dashed line).

\section{Conclusions}

The most stricking result of our simulation is that, during a probe orbital period, the sun-comet distance variations influence dust mass, flux and fluence much more than the probe-nucleus distance changes. This is a consequence of the small comet nucleus mass and, thus, of the non negligible probe orbital period with respect to the comet revolution time. The previous result supports our assumption of circular probe orbits. Moreover, only low eccentricity probe orbits and probe-nucleus distances of tens of nucleus size may offer stability against perturbations, due to the very probable asphericity of the nucleus. Models considering very eccentric keplerian probe orbits, passing very close to the nucleus, should include corrections during each revolution which are not predictable. Therefore, they appear less realistic than our conservative model based on circular probe orbits.

We must expect strong anisotropy of the dust ejection: it is very probable that the nucleus night side will release a negligible dust mass, whereas all the dust production will be confined in the sun side. Our results are mostly sensitive to the dust ejection anisotropy. Therefore, a detailed prediction of dust mass, flux and fluence versus the probe orbit anomaly, node and inclination is impossible without detailed information on the coma anisotropy patterns. It is unlikely that such data will be available before the ROSETTA probe will explore its target. The fit of the DIDSY data, coming from the fly-by of the 1P/Halley comet (Fulle et al. 1995), has shown that, on times comparable to the assumed probe orbital periods, a satisfying approximation of the ejection anisotropy pattern is given by a gaussian function of the zenithal 

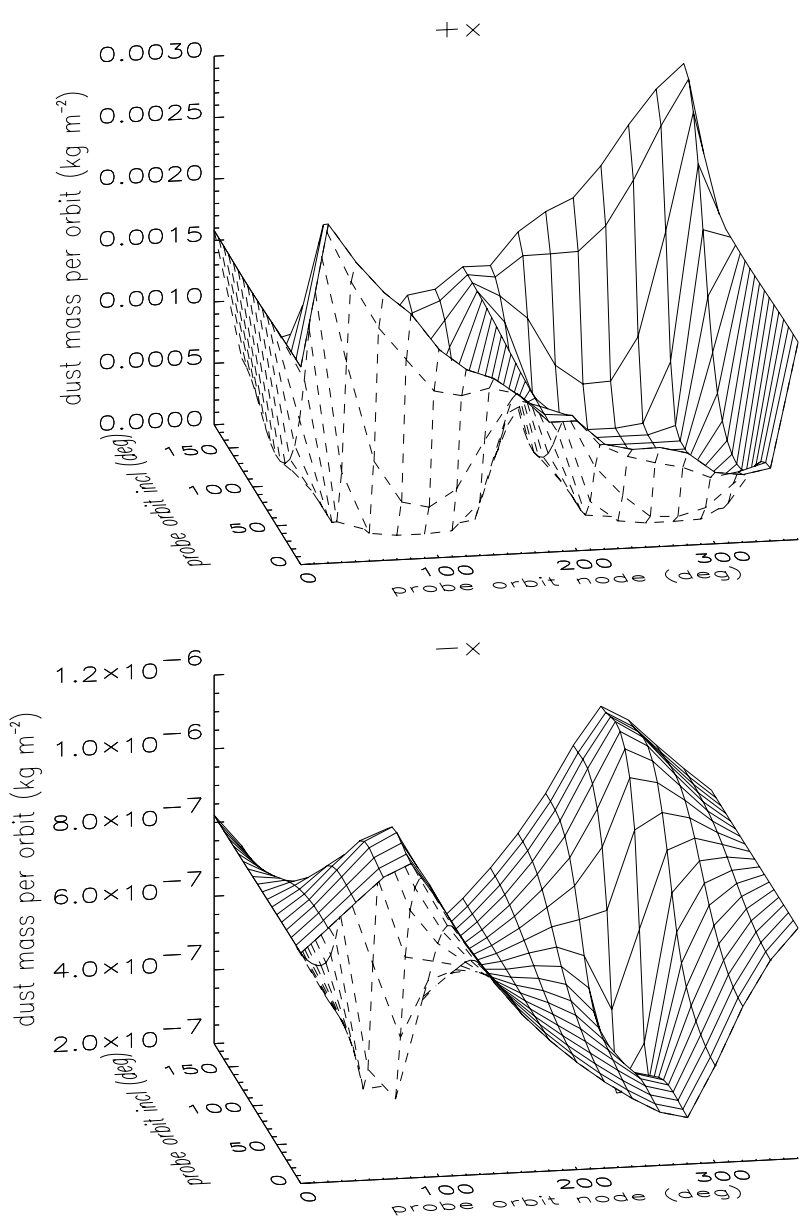

Fig. 19. Dust mass per unit surface collected during a probe orbit for $R=100 \mathrm{~km}$, starting sun-comet distance $r=$ $2.624 \mathrm{AU}, w=180^{\circ}$. Pointing directions: $+x$ and $-x$.

angle cosine. Planned observations of the ROSETTA target, 46P/Wirtanen, might provide information on the spin axis direction and coma morphology, which would allow us to improve the adopted model. For instance, if comet $46 \mathrm{P} /$ Wirtanen would display a fan shaped coma, due to active spots located close to the nucleus rotation pole, then the model would be improved by assuming the nucleus rotation axis as the direction of the highest dust production.

Observations of $46 \mathrm{P} /$ Wirtanen might allow us to better define poorly known parameters describing the comet dust environment. Some of these parameters have a predictable influence on the model results: they depend linearly on the gas loss rate or on the dust to gas ratio; so that changes of these parameters would imply a simple scaling of all the plots presented in this paper. Other parameters influence only some of the results. Changes of the dust bulk density would imply a scaling of the computed total masses and mass fluxes. Similarly, variations of fluences are produced by a dust size distribution power index change, which, instead, is ineffective on dust masses and
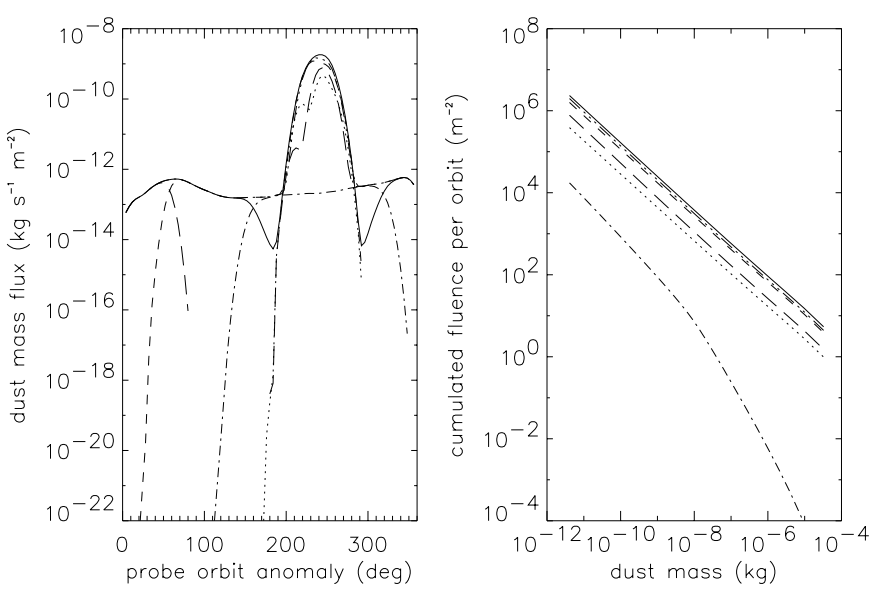

Fig. 20. Dust mass flux (left panel) and cumulated fluence (right panel) for $R=100 \mathrm{~km}$, starting sun-comet distance $r=2.624 \mathrm{AU}, w=180^{\circ}, n=120^{\circ}$ and $i=10^{\circ}$. Pointing directions: $+x$ (continuous line), $-x$ (dot dashed line), $+y$ (short dashed line), $-y$ (long dashed line), $+z$ (dotted line), and $-z$ (three dot dashed line).

mass fluxes, when considering the largest grains dragged by gas out of the nucleus. Other poorly known parameters strongly influence model results in a non predictable way. Information is lacking about the dust loss rate dependence on the sun-comet distance and the time of comet activity onset, that we have assumed to occur at aphelion. The gas ejection anisotropy influences the mass limit of grains dragged out by gas, which might be lower than the assumed values. This would imply cut-offs of the fluences at lower masses than those shown in the plots, mainly at large sun-comet distances, where the comet dust environment is unknown. Further observations are needed to constrain inner coma models which provide the dust ejection velocity: models predicting the dust flux which will be measured in-situ mainly depend on this poorly known quantity and on the dispersion around its most probable values.

The model presented in this work is a powerful tool for the prediction of results we can expect from ROSETTA experiments aimed at comet dust analysis. The reported conclusions about the dependence of dust mass, flux and fluence on the direction, in the probe reference frame, have a general validity. On the other hand, their absolute values have been obtained on the basis of the presently available best estimates of cometary environment parameters. These results will have to be updated as long as more refined information will be available on the target comet. With concern to the experiment on board ROSETTA expressely devoted to the dust flux monitoring, our results clearly indicate that direct grains will be collected only when the probe will cross the jets of the inner coma. During all other phases, the total mass of reflected grains, 
collected from both the directions perpendicular to the probe orbital plane, will be higher than that collected in the nucleus direction. According to our computations, these two directions will be the most suitable to monitor the reflected dust flux coming from the sun direction. To properly constrain the velocity determination of direct grains, an acceptance angle of about $40^{\circ}$ will be needed (Lamy et al. 1996). On the other hand, an acceptance angle of about $80^{\circ}$ is more efficient when measuring the flux of reflected grains. In this last case, according to our results, for $56 \%$ of randomly oriented probe orbits the flux received from both the directions perpendicular to the probe orbital plane is higher than that received from the nucleus direction. Most of these favourable orbits are not forbidden on the basis of the present mission plan. The collection of reflected grains is crucial to get information on dust ejected at sun-comet distances different from those characterizing the probe orbits. Finally, according to our model results, many probe orbits, similar to those considered in the present approach, will be needed to deposit a complete dust monolayer on the spacecraft and/or experiment surfaces pointing to the nucleus.
Acknowledgements. This work has been supperted by ASI, CNR and MURST research contracts. We thank Dr. J.F. Crifo for providing us the digital version of the output of his comet environment modelling and for the stimulating discussion. We thank Dr. J. Crovisier for the detailed review and for the suggested improvements to the paper.

\section{References}

Bar-Nun A., et al., 1993, ROSETTA-Comet Rendez-vous Mission, ESA Sci. 93, 7

Crifo J.F., 1991, in: Newburn R.L. Jr., Neugebauer M., Rahe J. (eds.), Comets in the Post-Halley Era. Kluwer, Dordrecht, p. 937

Crifo J.F., Rodionov A.V., 1996, Icarus (in press)

Finson M.L., Probstein R.F., 1968, ApJ 154, 327

Fulle M., Colangeli L., Mennella V., Rotundi A., Bussoletti E., 1995, A\&A 304, 622

Jorda L., Rickman H., 1995, PSS 43, 575

Lamy P.L., et al., 1996, Adv. Sp. Res. (in press)

McDonnell J.A.M., et al., 1987, A\&A 187, 719 